\title{
Analyses of Characteristics of Ring-shaped Electrostatic Meter
}

\author{
Dr Jianyong Zhang*, Dr Chuanlong Xu**, Dr Donglai Xu*, \\ Professor John Coulthard* Professor Shimin Wang** \\ *University of Teesside, United Kingdom \\ ** Southeast University, China
}

\begin{abstract}
This paper describes the characteristics of ring-shaped electrostatic pulverised fuel meters and their applications. At the University of Teesside, UK, electrostatic technique has been used to measure pulverised fuel flow concentration, velocity and mass flow rate under lean phase condition. The mathematic model has been developed to express the relationship between the root mean square (rms) value of meter's output voltage and solids mass flow rate. The effects of solids velocity and particle size on measurements have also been reflected in the model. Further more, the model presents the sensitivity variations over cross the sectional area of meter and along the pipe axis.

The paper also introduces the research carried out in the Southeast University in China, where the technique has been extended to measure dense phase flow of pulverised coal, which is common in gasification and blast furnaces.
\end{abstract}

Key words: Electrostatic, Pulverised fuel, air-solids two-phase flow, measurement

\section{Introduction}

The importance of correct fuel to air ratio in achieving clean and efficient combustion is well demonstrated. However the technology in coal fired power stations is complicated, where the fuel (coal) is pulverised and air-coal mixture is then pneumatically conveyed to the burners. Accurate measurement and control of the pulverised fuel (PF) mass flow rate inside each conveyor leading to burner is of critical importance for improving combustion efficiency, lowering the carbon content of the ash and reducing greenhouse gases and other emissions. The potential environmental and economic benefits from achieving effective PF flow measurement and thus combustion control are extremely significant. In a study by PowerGen, the economics of PF distribution control were analysed, suggesting that improving PF distribution with a combustion optimiser can provide simultaneous benefits in reduction of NOx emissions by $30 \%$ with instantaneous improvement of efficiency (Baimbridge P. 2001).

The use of coal as auxiliary fuels for injection into iron making blast furnaces is driven primarily by economic factors, especially the benefits derived from reducing the consumption of expensive coals for metallurgical coke making. In order to control the process, measuring pulverised coal flow is essential (Thomson A. et al, 2007) In pharmaceutical, food and cement making industries, the measurement of powder flow is of importance for safety, economic and operation purposes. 
However, achieving accurate and reliable measurement of powder flow in complex pneumatic conveying systems has proven technically challenging. A number of methods, based on different principles have been explored. The ringshaped electrostatic PF system (Zhang J., Coulthard J, 2005) is among those most promising methods (Miller D, 2000). The principle of the passive electrostatic technique for PF flow measurement is well known. In pneumatic conveying, electrical charging of the conveyed solids occurs. The primary sources of electrification are frictional contact charging between particles, charge transfer from one particle to another, and friction between particles and the conducting pipe wall.

The electrostatic meters described in this paper are dynamic electrostatic systems, in which the fluctuation level in the net charges carried by solids particles is measured to indicate solids mass flow rate. The charge fluctuation is regarded as a stationary and ergodic stochastic process, and the fluctuation level is considered to be proportional to the solids mass flow rate in lean-phase condition. This paper presents the test results which shows that this level increases with the increase of solids mass flow rate.

The density of single phase flow is regarded as constant. However in air-solids two-phase flow, inhomogeneous solids distribution is a common phenomenon, which means that PF may not be uniformly distributed in the pipe, often concentrating to form a 'roping flow' so that it becomes highly concentrated locally whilst elsewhere there is very little solid (Huber N, Summerfield M, 1994, Frank T. 2007). This non-uniform flow pattern presents real difficulties to achieving accurate measurement as flow profiles varies with the pipe runs and loading. The meter's reading is dependent on flow profiles (Wang Shimin, et al, 2003, Zhang J., Coulthard J. 2005). To overcome the above problems and achieve accurate measurement of such flow, the metering system with uniform spatial sensitivity is ideal. This paper presents the analysis results of the spatial sensitivity of the ring-shaped electrostatic meter, which is the step forward towards achieving a uniform spatial sensitivity.

The velocity and size of solids particles affect the electrical charges carried by solids due to the mechanism of electrification. Under a given solids mass flow rate, the net charge carried by solids varies with the velocity and the particle size. Since the average velocity can be measured independently, e.g. using cross-correlation method, theoretically, this effect can be estimated or compensated. This paper demonstrates the effect of the velocity on the measurement. If the mass flow rate and the velocity are known parameters, the particle size information can be obtained from the charges induced on an electrode. This will also be discussed in this paper.

In a pneumatic conveying system, the charges carried by particles depend on many factors, such as humidity, chemical property of solids material, solids velocity, particle size and etc. Therefore the electrostatic meter does not indicate the absolute mass flow rate, it measures the relative mass flow rate, i.e. the ratio between the solids mass in each individual pipe to the total mass in a group of pipes emerging from a mill in power stations. 
A ring-shaped electrostatic meter comprises metal rings, which are insulated from and mounted flush with the earthed pipe. It is easy to manufacture, maintenance free, non intrusive to the flow and robust.

\section{Basic mathematic model of a ring shaped electrode}

The charge induced on a ring-shaped electrode by a single particle having unity charge has been modelled (Cheng $\mathrm{R}$ 1996), which was based on electrostatic theory and calculated using the finite element method (FEM). As shown in Fig.

1, the charge $Q$ induced on the inner surface of the electrode due to a particle of unity point charge inside the pipe was calculated using following expression;

$$
Q=\int_{S} \sigma \cdot d s
$$

where $\sigma$ is the surface density of the charge induced on the electrode, and $\mathrm{S}$ is entire inner surface area of the electrode. The FEM analysis results were curve fitted to the following equation.

$$
\mathrm{Q}=\mathrm{Ae}^{-k^{2} x^{2}}
$$

where $A$ and $k$ are two constants that are functions of the width $W$ of the electrode and the radius $r$ from the central axis of the electrode towards the pipe wall. The variable $x$ denotes the distance along the pipeline from the centre of the electrode cross section as illustrated in Fig. 1. The quantity $Q$ is the charge induced on a metallic electrode ring from a particle carrying unity point charge.

The sensitivity of a ring-shaped electrode versus the location of a charged particle illustrated in Fig. 2 based on equation (2), $A=0.170, k=-0.01056$, for $\mathrm{W} / \mathrm{R}=1 / 5, \mathrm{r} / \mathrm{R}=0.5$. It can be seen that the sensitivity is a function of the radius $\mathrm{r}$ for a given electrode width W. Fig. 3 and Fig. 4 show the spatial sensitivity of a ring-shaped electrode and the relative AC signal.

The sensing volume, shown in Fig.5, indicates that the sensor can 'detect' streams of particles moving at different distances on different axes. It can sense particles at greater distances along an axial line closer to the pipe central axis than particles moving closer to the pipe wall. Obviously, the sensing volume is significantly different to the physical volume determined geographically by the electrode width $\mathrm{W}$ and the cross sectional area of the electrode.

In the Southeast University, the modelling has been modified, resulting in the following equation,

$$
S(z)=a e^{-b z^{2}}+c e^{-d z^{2}}
$$

Where $\mathrm{S}(\mathrm{z})$ stands for sensitivity along pipe line, $\mathrm{z}$ is the coordinate along pipe line. Fig. 6 shows the spatial sensitivity based on equation (3). The similarity between these two models is obvious although the electrode is not directly 
exposed to the flow in the Southeast University's design, in which, the inner surface of electrode was lined with a quartz tube.

In equation (3), $z=0$ is at the edge of the sensing volume, whilst in equation (2), $x=0$ is at the middle of the sensing length along the pipe axis.

Fig.7 depicts the sensitivity of a ring-shaped electrode against radius $r$ at different position $\mathrm{z}$ along pipe line. This is obtained based on the Southeast University's Model.

If we take the difference in definitions between $\mathrm{x}$ and $\mathrm{z}$ into account, it is clear that Fig.5 and Fig. 7 describe the same phenomenon that spatial sensitivity varies with radius $r$ at different position along pipe axis.

\section{Model of induced signal to stream of charged particles}

A ring-shaped stream having inner radius $r$ and outer radius $r+\Delta r$ can represent a roping flow due to the symmetrical property of the ring-shaped electrode. Based on the principle of superposition of electrostatic fields, the response of a ring-shaped electrode to this stream can be expressed as follows according to equation (2),

$$
Q_{N E T}^{r}=\sigma_{p} \cdot \pi \cdot D_{P}^{2} \cdot N \int_{L_{x}} A(r, W) e^{-k(r, W) x^{2}} d x
$$

where $\mathrm{A}$ and $\mathrm{k}$ in equation (2) are replaced with $\mathrm{A}(\mathrm{r}, \mathrm{W})$ and $\mathrm{k}(\mathrm{r}, \mathrm{W})$ to represent their dependences on electrode width and position of the stream with respect to the central axis (Zhang J., Coulthard J. 2005).

If we consider this roping particle stream wave travelling at velocity $V$ as a delta function, i.e.

$$
Q_{N E T r}^{P}=\sigma_{p} \cdot \pi \cdot D_{P}^{2} \cdot N(t)=\delta\left(t-\frac{x}{V}\right)
$$

at any given time, at only one point along $x$ direction where $Q_{N E T r}^{P}$ exists, This point is $\mathrm{x}=\mathrm{V}^{*} t$. Hence Equation (4) becomes

$$
Q_{N E T}^{r}=h_{r d}(t)=A(r, W) e^{-k(r, W) V^{2} t^{2}}
$$

This equation describes the dynamic response of a ring-shaped electrode to a unit impulse of net charge carried by solid particles at radius $\mathrm{r}$. The variable $h_{r d}(\mathrm{t})$ is sensitive to the stream spatial position..

Applying Fourier transformation to Equation (6), the spatial frequency response characteristic of a ring-shaped electrode to a unit impulse of net charge carried by particles is,

$$
H_{r}(\varpi)=\int_{-\infty}^{\infty} h_{r d}(t) e^{-j \varpi t} d t=\int_{-\infty}^{\infty} A(r, W) e^{-k(r, W) V^{2} t^{2}} e^{-j \varpi t} d t=\frac{A(r, W) \pi^{\frac{1}{2}}}{V \sqrt{k(r, W)}} e^{-\frac{\varpi^{2}}{4 V^{2}} \frac{1}{k(r, W)}}
$$




\section{Effect of velocity on measurement of solids mass flow rate}

From equation (7), it is evident that the velocity affects the frequency characteristics of the electrode and thus the entire metering system. The detailed analysis of the frequency spatial characteristics of electrode and ring-shaped electrostatic sensors can be found in elsewhere (Zhang J., Coulthard J., 2005), (Cheng R. 1996), (Zhang J. 2004), (Xu C., 2005). Fig. 8 demonstrates that the frequency bandwidth and amplitude peak position of the transfer function of a ring-shaped electrostatic sensor increases with velocity.

In the Southeast University, the investigations into effects of velocity on the flow measurement as shown in Fig 9 have led to a novel method for solids velocity measurement based on signal frequency spectrum analysis.

A complete metering system comprises the electrode and the signal conditioning circuit. Thus the transfer function of a sensor can be obtained from the integration of $\mathrm{H}_{\mathrm{r}}(\omega)$, i.e. $\int_{0}^{R} H_{r}(\omega) d r$ multiplied by the transfer function of the circuit. For a dynamic measurement system, the root mean square value of meter's output voltage can be related to its power spectral density function according to Parseval's formula. The square value of the system output voltage is given by equation (8) assuming that the solids concentration is proportional to the net charges carried by particles.

$$
U_{r m s}^{2} \propto \frac{\operatorname{con}^{2}}{\varpi_{\max }} V
$$

where Con is the concentration of flow, $\mathrm{V}$ is the solids velocity, $\omega_{\max }$ is the maximum frequency of band-limited flow noise.

From this equation, it can be seen that the rms value of the output voltage of a sensor is proportional to solids concentration and the square root of the velocity. Thus, in theory, the effect of velocity on concentration measurement can therefore be removed. The solids mass flow rate is the product of solids concentration, average solids velocity and pipe cross sectional area, thus the solids mass flow rate can also be measured based on equation (8).

It also shows that $U_{\text {rms }}$ is linear with solids concentration or solids mass flow rate under a given velocity. Fig.10. and Fig.11 provide laboratory test results supporting the above conclusions.

\section{Effect of Particle size}

Cheng (Cheng R., 1996) described the principle of measuring particle size based on signal bandwidths. For a given electrode geometry, the signal waveform and the corresponding signal bandwidth are fundamentally dependent upon the time that particles remain within the sensing volume. Therefore, larger particles remain within the sensing volume for a 
longer time corresponding to a narrower signal bandwidth, than that produced by smaller particles moving at the same speed. This has been confirmed by modelling carried out at the laboratory of the Southeast University as illustrated in Fig 12.

This has been investigated at the University of Teesside. The project was funded by ECSC (European Coal and Steel community) (Research Report, 2004), Fig. 13 shows the autocorrelation curves of signals of sensor output under different particle sizes.

It can be seen that for the smaller particles, the autocorrelation curve becomes narrower due to higher frequency bandwidth of signal. But the resolution is poor for narrow size distributions. This method may be useful in coal preparation where large and small sizes of coal need to be distinguished.

Another on-line particle size measurement method investigated is based on the assumption that surface charge density $\sigma_{p}$ of particles is constant. It gives that the charge carried by a particle is proportional to its surface or $\mathrm{D}^{2}$ for spherical particles, where D is particle diameter (Zhang J., Coulthard J., 2006)

Under the above assumptions, Equation (8) can be modified to include the particle size information, which is

$$
U_{r m s}^{2} \propto \frac{\operatorname{con}_{r m s}^{2}}{D^{2} \varpi_{\max }} V
$$

Fig. 14 shows the test results obtained in a 40mm diameter rig in Casella CRE Energy in Gloucestershire, UK. The material was coal powder. The size range covered $75 \mu \mathrm{m}$ to $1 \mathrm{~mm}$. Fig. 15 was drawn based on laboratory tests results at the University of Teesside, the materials was sand, the particle size was in the range of $280 \mu \mathrm{m}$ to $900 \mu \mathrm{m}$. The results confirmed that the signal rms value decreases with particle size under constant solids mass flow rate and velocity.

\section{Conclusions}

Although electrostatic meters provide only relative solids mass flow rate and concentration information, it has been successfully used in coal fired power stations for split and velocity measurement, and used as diagnostic tool to identify poor conveying conditions.

Theoretical and experimental investigations into spatial sensitivity, effects of particle size and velocity on flow measurement have played important roles in improving the performance of meters and to extend its applications. For example, in the University of Teesside and the Southeast University, some of research findings have been applied in different aspects such as an innovative method of flow velocity measurement based on frequency spectrum analysis and particle size indication using electrostatic meter.

The research in improving spatial sensitivity and solids mass flow measurement based on Fussy logic are ongoing activities in these two universities. 
The electrostatic meters with ring shaped electrodes are non-intrusive, robust, maintenance free and low cost. The electrostatic method will remain as a practical and promising approach in metering air-solid two-phase flow.

\section{References}

Baimbridge P., ECSC Project 7220-PR-050:Measurement and control techniques for improving combustion and reducing emissions from coal fired plant, Boiler integration Study, 02/2001.

Cheng R., A study of electrostatic pulverised fuel meters, Ph.D thesis, University of Teesside, U.K. 1996.

Frank Thomas, Lagrangian prediction of pulverized fuel flow in bifurcators and trifurcators, http://www.imech.tuchemnitz.de/mpf, 2007.

Huber N, Summerfield M, Characterisation of the cross-sectional particle distribution in pneumatic conveying system, Powder technology, Vol.79, 1994

Miller Dave, et al, Technology status review report: PF flow measurement and control methods for utility boilers, December 2000 .

Research final report, On-line Measurement of particle size in fine coal transport systems, Project ECSC-7220-PR-105, 31, July 2004.

Thomson Angus, Zulli Paul, McCarthy Malcolm and Horrocks Keith, Pulverised coal injection in iron making blast furnaces, The Australian coal review, Oct. 1996, pp42-46.

Wang Shimin, et al, Advanced measurement and diagnosis technology applied in coal-fired utility boilers, Proceedings of $5^{\text {th }}$ International symposium on coal combustion, Nanjing, China, November 23-26, 2003, paper No. 14.

$\mathrm{Xu}$ ChuanLong, Gas-solids two phases flows parameters and particle electrostatic measurement, Ph D thesis, Souheast University, Nanjing, China, Dec. 2005.

Zhang Jianyong, Coulthard John, Cheng Ruixue, Keech Ray, Measuring and controlling pulverized fuel on coal-fired plant, Journal of clean air, vol 6, No.1 pp 25-35, 2005

Zhang Jianyong, Coulthard John, Theoretical and experimental studies of the spatial sensitivity of an electrostatic pulverised fuel meter, Journal of electrostatics, Vol.63, issue 12, Oct 2005

Zhang Jianyong, A study of an electrostatic flow meter, Ph D thesis, April 2002, University of Teesside, U.K.

Zhang Jianyong, Coulthard John, On-line indication of variation of particle size using electrostatic PF meters, The proceedings of the 31th international technical conference on coal utilization and fuel systems, May 21-25, 2006, Clearwater, Florida, USA 

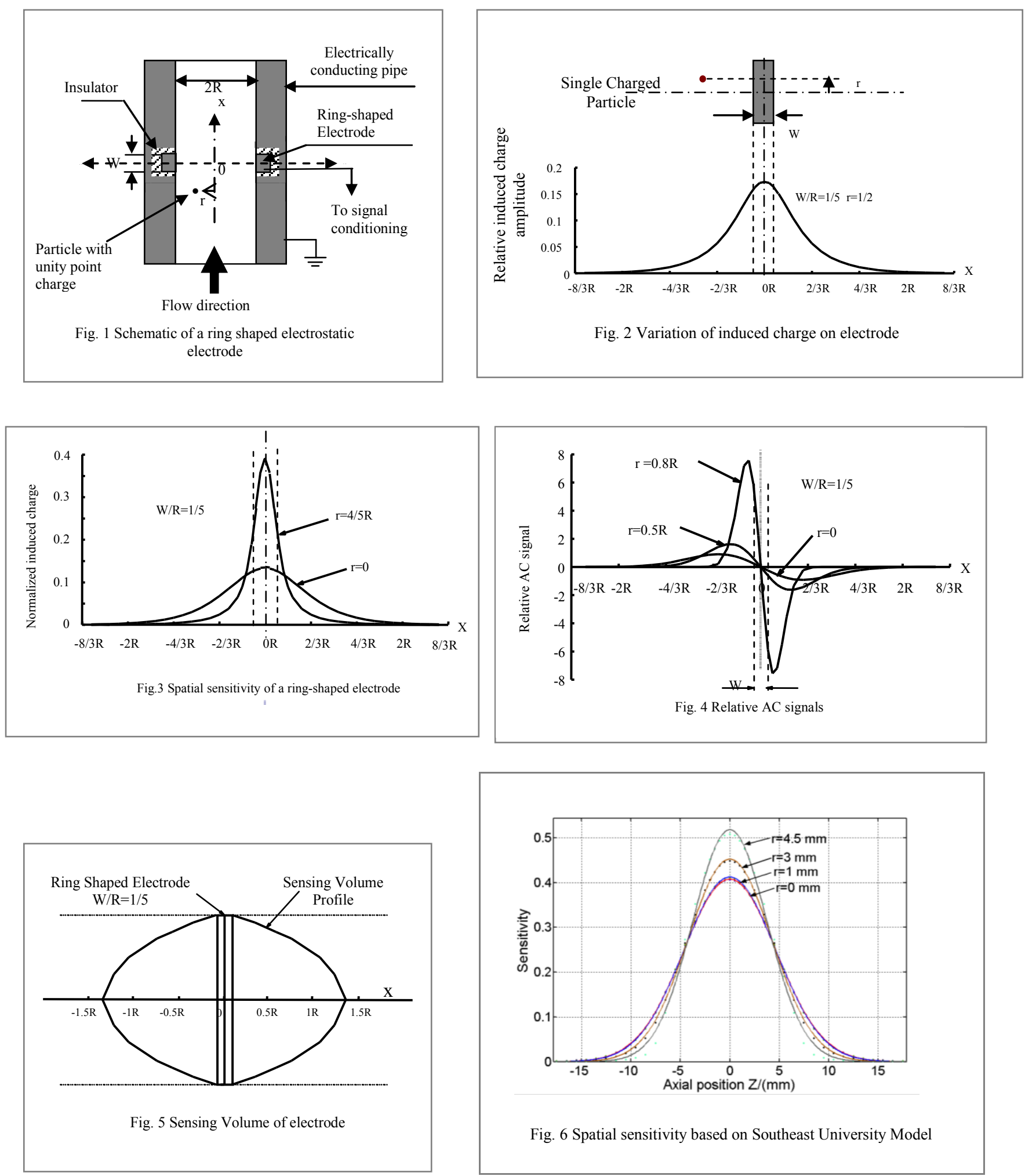

Fig. 6 Spatial sensitivity based on Southeast University Model 


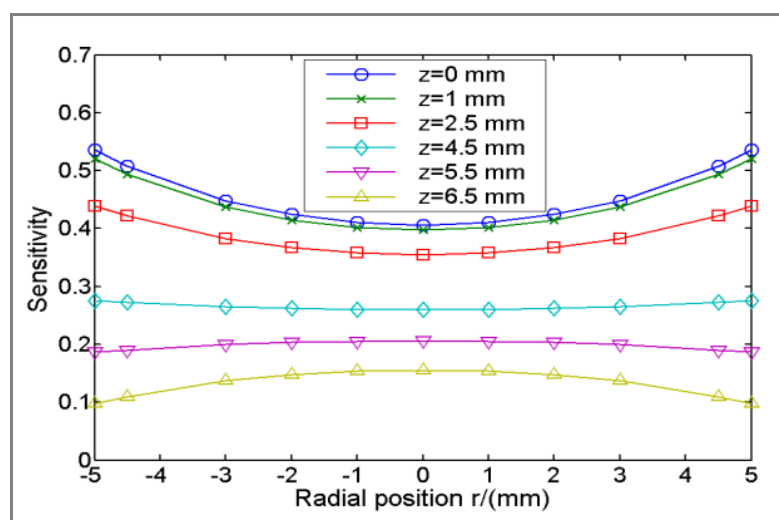

Fig. 7 Sensitivity versus $r$ at given $z$

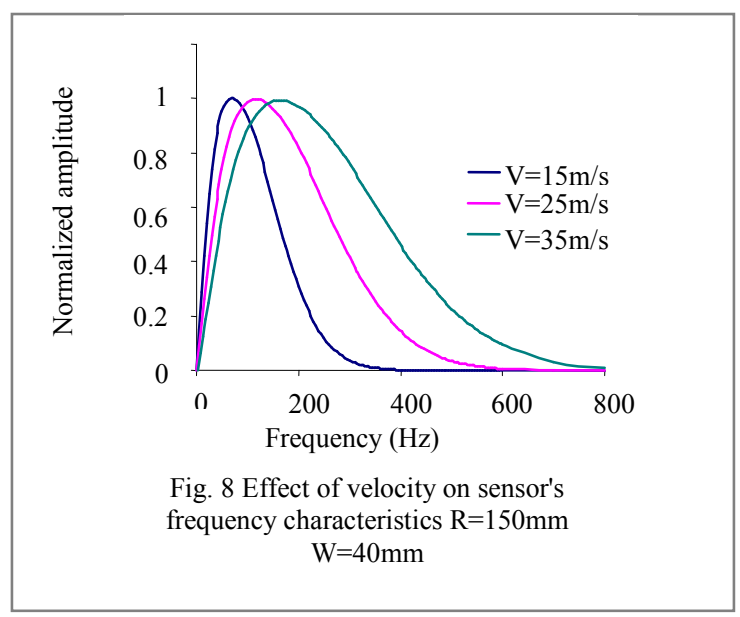

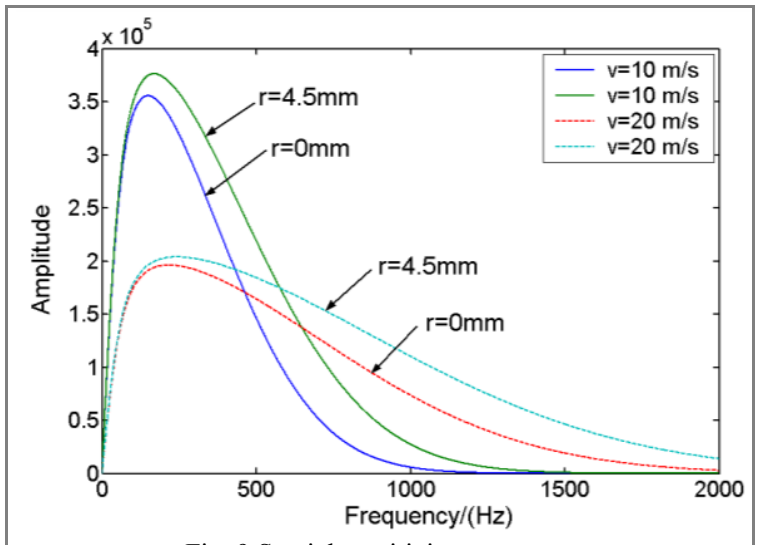

Fig. 9 Spatial sensitivity

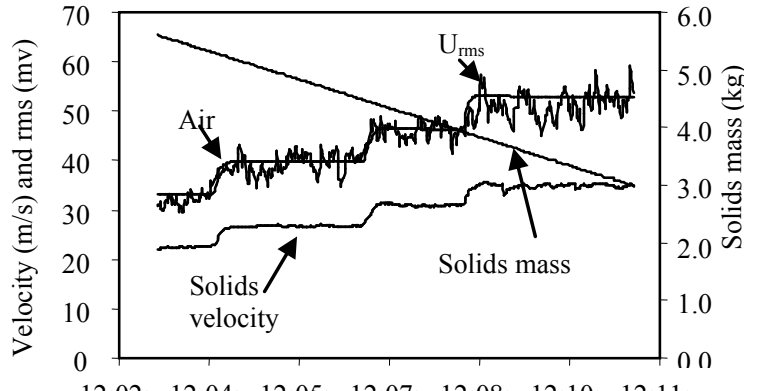

12:02: 12:04: 12:05: 12:07: 12:08: 12:10: 12:11:

Fig 10. Variation in $\mathrm{U}_{\mathrm{rms}}$ with solids velocity under constant mass flow rate

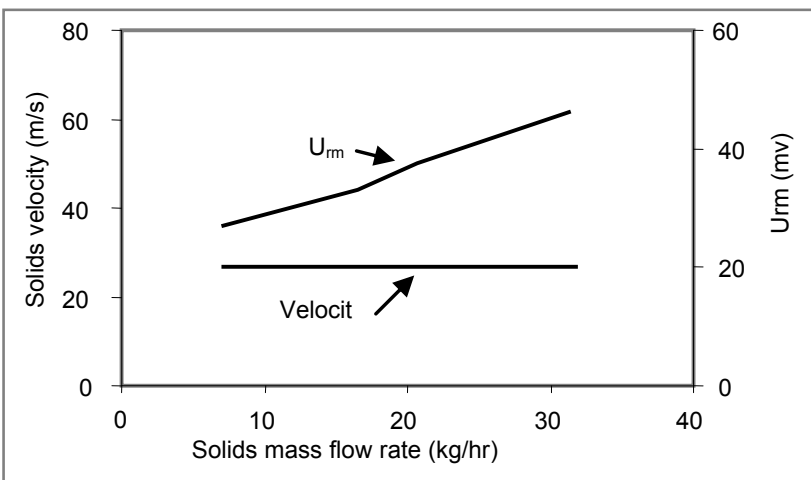

Fig. $11 \mathrm{U}_{\mathrm{rms}}$ variation with solids mass flow rate under constant velocity

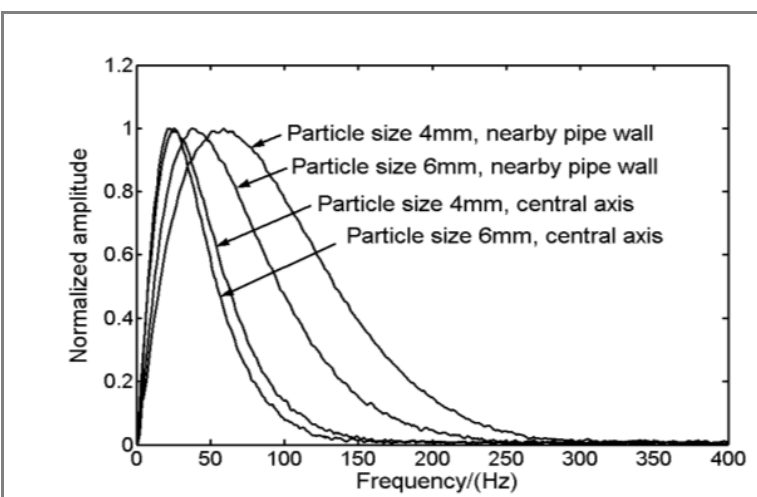

Fig. 12 Effect of particle size on signal frequency spectrum 


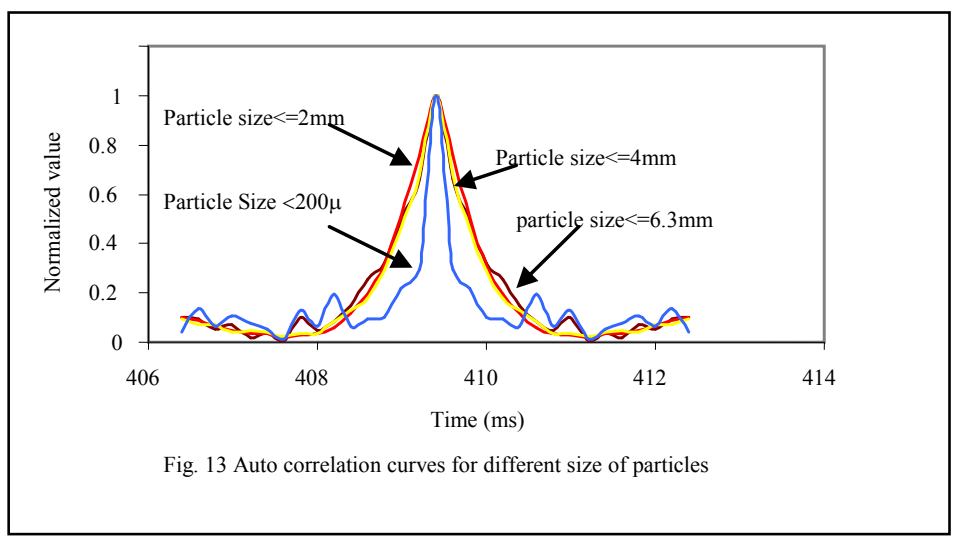

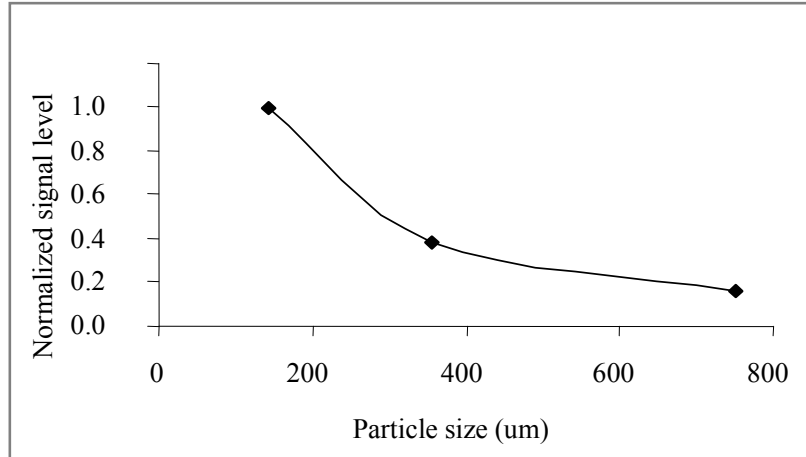

Fig. $14 U_{\text {rms }}$ versus particle size

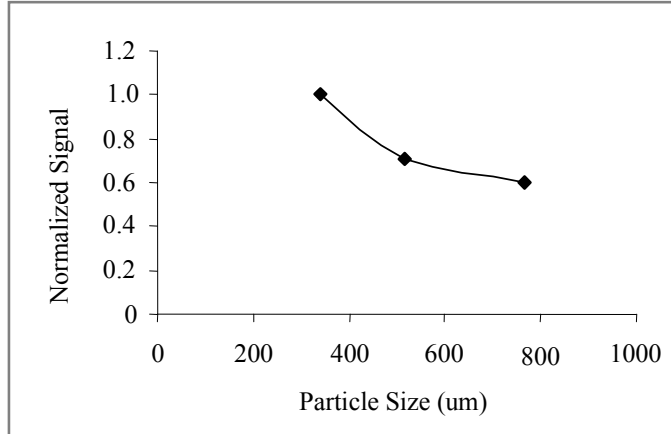

Fig.15 Particle size measurement test results using sand 
Communication details of the designated Author:

Dr. Jianyong Zhang

School of Science and Technology

University of Teesside

Middlesbrough, TS1 3BA

U.K.

Tel: 0044(0)1642342546

Fax:0044(0)1642342401

-mail: j.zhang@tees.ac.uk 